Internist 2020 $61: 254-260$

https://doi.org/10.1007/s00108-020-00748-6

Online publiziert: 6 . Februar 2020

(c) Der/die Autor(en) 2020

\section{Redaktion}

E. Märker-Hermann, Wiesbaden

M. Wehling, Mannheim

\author{
W. N. Löscher' $\cdot$ B. Iglseder ${ }^{2}$ \\ ' Universitätsklinik für Neurologie, Medizinische Universität Innsbruck, Innsbruck, Österreich \\ ${ }^{2}$ Universitätsklinik für Geriatrie der Paracelsus Medizinischen Privatuniversität Salzburg, Christian- \\ Doppler-Klinik, Salzburg, Österreich
}

\title{
Polyneuropathie im Alter
}

zierter Vibrationssinn, besonders distal an den Beinen, wurde bei $71 \%$ einer gesunden Altersgruppe nachgewiesen [43].

Zusätzlich führen diese Alternsvorgänge des PNS in teils kausalem Zusammenspiel mit der Abnahme der Muskelmasse (Sarkopenie) zu einem Verlust an Muskelkraft und Gleichgewicht. Dieses Zusammenspiel neuronaler und muskulärer Faktoren als Ursache der Muskelschwäche im Alter fand unter dem Terminus Dynapenie Eingang in die Literatur [8]. Daraus resultieren - zusätzlich beeinflusst von zentralen Alterungsphänomenen - funktionelle Einschränkungen wie reduzierte Mobilität und erhöhtes Sturzrisiko [5, 37].

\section{》) Die PNP wirkt sich deutlich auf Selbsthilfefähigkeit, Alltags- aktivitäten und Sturzrisiko aus}

In der Rotterdam-Studie wurde bei älteren Probanden mit einem Durchschnittsalter von 71 Jahren der Zusammenhang zwischen Alltagsfähigkeiten, Stürzen und PNP untersucht. Bei Vorliegen einer PNP fand sich ein deutlich erhöhtes Sturzrisiko (Odds Ratio [OR] 1,87; $95 \%$ Konfidenzintervall [KI] 1,10-3,16), in diesem Kontext war das Risiko, im Rahmen des Sturzes eine Fraktur zu erleiden, besonders akzentuiert (OR 3,35; 95\%-KI 1,02-10,97). Zudem boten die Betroffenen schlechtere Ergebnisse bei basalen und instrumentalen Aktivitäten des täglichen Lebens wie Gehvermögen, Mobilität oder Einkaufen. Auch Gehgeschwindigkeit, Kadenz und Tandemgang als Parameter der Ganganalyse fielen bei Vorliegen einer PNP schlechter aus. Die
PNP hat somit deutliche Auswirkungen auf Selbsthilfefähigkeit, Aktivitäten des täglichen Lebens und Sturzrisiko [16].

\section{Epidemiologie und Ätiologie - Ursachen im Alter}

Die Inzidenz von PNP beträgt in den Niederlanden bei Personen über 18 Jahre 77/100.000, nimmt aber mit dem Alter deutlich zu: Von $\sim 60 / 100.000$ bei 50 - bis 54-Jährigen auf $\sim 300 / 100.000$ bei 75 - bis 79-Jährigen [41]. Eine große Metaanalyse ergab eine Prävalenz von $1 \%$ für die Gesamtbevölkerung, die auf $3 \%$ bei $>55$-Jährigen und auf $7 \%$ in der älteren Population steigt [17]. Das gehäufte Auftreten im Alter beschrieb Oppenheim bereits 1893 als vorwiegend durch sensible Reizerscheinungen gekennzeichnete „Polyneuritis senilis“ [28].

\section{》) Eine Besonderheit des Alters ist der hohe Anteil an Neuropathien ohne eindeutige Ursache}

Die Ursachen von Neuropathien sind auch im Alter mannigfaltig: Neben metabolischen, immunvermittelten, hereditären, toxischen und infektiösen Ätiologien treten PNP im Rahmen von Systemerkrankungen auf. Eine Besonderheit des Alters ist der hohe Anteil an Neuropathien ohne eindeutige Ursache, man spricht von kryptogenen oder chronischen idiopathischen Neuropathien. Erwartungsgemäß nimmt im Alter die Inzidenz der diabetischen PNP zu (etwa $30 \%$ der Fälle), während etwa immun- 
Hier steht eine Anzeige.

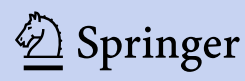


Tab. 1 Ursachen von Neuropathien be

785 Patienten über 65 Jahre. (Nach [27])

\begin{tabular}{|l|l|}
\hline Ursache & $\begin{array}{l}\text { Häufigkeit } \\
\text { (\%) }\end{array}$ \\
\hline Idiopathisch & 35,4 \\
\hline Diabetes & 18,8 \\
\hline Immunologisch & 7,9 \\
\hline Toxisch & 7,4 \\
\hline Entzündlich & 6 \\
\hline Hereditär & 3,4 \\
\hline Infektiös & 3 \\
\hline Alkohol & 2,9 \\
\hline Monoklonale Gammopathie & 2,9 \\
\hline unklarer Signifikanz & 2,5 \\
\hline Hämatologisch & 2 \\
\hline Makroglobulinämie & 2 \\
\hline Karzinom & 2 \\
\hline $\begin{array}{l}\text { Verschiedene metabolische } \\
\text { Erkrankungen }\end{array}$ & 1,9 \\
\hline Niereninsuffizienz & 1 \\
\hline Primäre Amyloidose & 1 \\
\hline Verschiedenes & \\
\hline
\end{tabular}

vermittelte Neuropathien anteilsmäßig deutlich abnehmen [41].

So fand eine retrospektive Untersuchung an 785 Patienten über 65 Jahre eine kryptogene Neuropathie in $35,4 \%$ und eine diabetische Neuropathie in $18,8 \%$ der Fälle (• Tab. 1; [27]). Ähnliches wird auch in anderen Studien berichtet [15, 41].

Angesichts der Polypharmazie im Alter sollten medikamenteninduzierte PNP nicht übersehen werden. Die Liste der PNP-verursachenden Medikamente ist lang, zu erwähnen sind vor allem Chemotherapeutika, einzelne Virostatika und Antibiotika sowie Statine und Amiodaron [21].

\section{Besonderheiten der klinischen und apparativen Diagnostik im Alter}

Die altersassoziierten Veränderungen des PNS können auch bei gesunden alten Menschen zu Symptomen und Zeichen führen, die denen einer Neuropathie entsprechen [43]; so berichten $20 \%$ der gesunden alten Menschen über Parästhesien oder Hypästhesien, 28 bzw. $21 \%$ über Krämpfe oder Steifigkeit, beides vorwiegend in den Beinen. In der klinischen Untersuchung gesunder alter Menschen wurden ein abnormer Vibrationssinn in $71 \%$ und ein fehlender Achillessehnenreflex in 38\% der Fälle gefunden. Abnormer Vibrationssinn und fehlender Achillessehnenreflex werden bei Gesunden ab dem 60. Lebensjahr beobachtet [44]. Altersabhängige Normwerte für die Bestimmung des Vibrationssinns mit der Rydel-SeifferStimmgabel wurden publiziert [26].

\section{》) Orthopädische Ver-}

änderungen können die Gangunsicherheit einer Neuropathie vortäuschen

Auch orthopädische Veränderungen wie Arthrosen oder Hallux valgus können die Gangunsicherheit einer Neuropathie vortäuschen, ebenso wie die altersbedingte Verschlechterung von Gleichgewicht, Sehen, Hören und zentraler Regulation der posturalen Kontrolle. All dies muss bei der Diagnose einer sensiblen Neuropathie im Alter bedacht werden. In der klinischen Untersuchung von Stand und Gang sind das erhöhte Sturz- und Frakturrisiko alter Menschen zu beachten, besonders bei Wendemanövern und unter Ausschaltung der optischen Kontrolle bei geschlossenen Augen.

Elektrophysiologische Untersuchungen werden vor allem zur pathophysiologischen Beurteilung von Neuropathien, aber auch zu deren Objektivierung eingesetzt. Es hat sich jedoch gezeigt, dass sich die meisten Parameter der Elektroneurographie mit dem Alter verändern, vor allem nehmen die Amplituden der sensiblen, aber auch der motorischen Potenziale ab [39, 45]. Da die Diagnose einer axonalen Neuropathie, die mit $\sim 90 \%$ im Alter die bei Weitem häufigste Form darstellt [27], zu einem großen Teil auf der Reduktion der Amplituden dieser Potenziale beruht, kann die elektrophysiologische Objektivierung erschwert sein.

\section{Wichtiges zu einzelnen Neuropathien}

Das klinische Bild, der Krankheitsverlauf und die Therapie von Neuropathien sind im Alter und in jüngeren Jahren ähnlich. Allerdings ist die Prognose im Alter häufig schlechter, wie etwa beim Guillain-Barré-Syndrom [40].

\section{Chronische idiopathische axonale Polyneuropathie/kryptogene Neuropathie}

Die chronische idiopathische axonale PNP (CIAP) ist die häufigste Neuropathie im Alter [41]. Klinisch ist sie definiert durch eine relativ milde und nur sehr langsam progrediente Klinik vor allem in Form distaler Sensibilitätsstörungen an den Beinen, einer milden sensiblen Ataxie und neuropathischer Schmerzen [34]. Paresen fehlen, erlaubt ist per definitionem nur eine geringe Parese der Zehenextensoren. Elektrophysiologisch handelt es sich um eine axonale Neuropathie.

Bekannte Ursachen von Neuropathien müssen vor Diagnose einer CIAP ausgeschlossen werden [34, 47]. Zu beachten ist, dass vor allem Ursachen von Neuropathien mit ähnlichem Phänotyp abgeklärt werden, und nicht jede nur erdenkliche Ursache. In erster Linie müssen metabolische, toxische und systemische Faktoren ausgeschlossen werden. Es hat sich gezeigt, dass die CIAP häufiger mit einem metabolischen Syndrom assoziiert ist [42], was unter Umständen einen therapeutischen Ansatz bietet. In der Betreuung der betroffenen Patienten ist es entscheidend zu betonen, dass die Erkrankung nur langsam progredient ist und mild verläuft.

\section{Lumbosakrale Radikuloplexus- neuropathie}

Im medianen Alter von 65 bis 70 Jahren kann die lumbosakrale Radikuloplexusneuropathie (LRPN) auftreten [11, 12]. Es kommt zu akut einsetzenden heftigen Schmerzen, die von lumbal zur ventralen Seite eines Oberschenkels ziehen. Rasch - innerhalb von Wochen bis Monaten entwickeln sich Lähmungen und Atro- 
phien der Beckengürtel- und Oberschenkelmuskeln, begleitet von einem teilweise deutlichen Gewichtsverlust. Sensibilitätsstörungen stehen im Hintergrund. Die Erkrankung beginnt in der Regel einseitig, breitet sich auf die andere Seite aus, bleibt aber meist asymmetrisch. Zum Diagnosezeitpunkt sind die meisten Betroffenen nicht selbstständig gehfähig. Die Erkrankung verläuft monophasisch, eine langsame Besserung beginnt in der Regel ab dem sechsten Monat.

Männer erkranken häufiger. Die Erkrankung kann idiopathisch oder im Rahmen eines Diabetes als diabetische LRPN, früher auch diabetische Amyotrophie genannt, auftreten. Die Differenzialdiagnose zu radikulären Ausfällen kann schwierig sein und bildgebende Verfahren notwendig machen. Die Zellzahl im Liquor ist unauffällig, das Eiweiß kann leichtgradig erhöht sein. Die Prognose ist variabel, Schmerzen können wochenlang anhalten. Da die histologische Untersuchung von Nervenbiopsaten eine epineurale Vaskulitis zeigte [11], sind Behandlungsversuche mit intravenösen Steroiden oder Immunglobulinen vertretbar.

\section{Polyneuropathien bei mono- klonalen Gammopathien unklarer Signifikanz}

Im Alter finden sich vermehrt Paraproteinämien (monoklonale Gammopathien). In seltenen Fällen treten diese im Kontext hämatologischer Erkrankungen wie des multiplen Myeloms oder Morbus Waldenström auf, meist findet sich allerdings eine monoklonale Gammopathie unklarer Signifikanz (MGUS). Diese altersabhängige Veränderung tritt bei 5,3\% der $>70$-Jährigen und bei $10 \%$ der $>80$ Jährigen auf und ist bei $10 \%$ der Patienten mit kryptogener Neuropathie nachweisbar [32]. Das Risiko der Progression zu einer hämatologischen Erkrankung beträgt etwa $1 \%$ pro Jahr, unterscheidet sich aber zwischen Immunglobulin-M(IgM)und Non-IgM-MGUS [22].

MGUS sind mit verschiedenen Phänotypen von Neuropathien assoziiert. Genauer mit

- der distal symmetrischen demyelinisierenden Neuropathie (DADS),

Internist 2020 -61:254-260 https://doi.org/10.1007/s00108-020-00748-6

(c) Der/die Autor(en) 2020

W. N. Löscher · B. Iglseder

\section{Polyneuropathie im Alter}

\section{Zusammenfassung}

Das periphere Nervensystem ist im Laufe des Alternsprozesses Veränderungen unterworfen. So kommt es unter anderem zu einer Abschwächung von Muskeleigenreflexen und Propriozeption. Davon abzugrenzen sind Polyneuropathien als krankhafte Veränderungen des peripheren Nervensystems. Die jährliche Inzidenz von Polyneuropathien wird auf 118/100.000 geschätzt, die Prävalenz liegt bei etwa $1 \%$, für ältere Populationen werden $7 \%$ angegeben. Die Ursachen sind vielfältig und ähnlich den Ursachen von Neuropathien des jüngeren Alters: Neben metabolischen, immunvermittelten, hereditären, toxischen und infektiösen Ätiologien können Polyneuropathien Ausdruck von Systemerkrankungen sein. Entsprechend der Altersverteilung der verschiedenen Ursachen sind Neuropathien im Zusammenhang mit Diabetes, monoklonalen Gammopathien und Malignomen im Alter häufiger. Allerdings nimmt der Anteil der kryptogenen Neuropathien, also ohne eindeutige Ursache, mit dem Alter zu. Bei alten Menschen führen Polyneuropathien zu einer zusätzlichen Beeinträchtigung der Mobilität und einem erhöhten Sturzrisiko, was auch die Abklärung funktioneller Fähigkeiten erforderlich macht.

\section{Schlüsselwörter}

Altern · Peripheres Nervensystem · Chronische idiopathische axonale Polyneuropathie . Diabetische Neuropathien · Monoklonale Gammopathie unklarer Signifikanz

\section{Polyneuropathy in older individuals}

\section{Abstract}

The peripheral nervous system is subject to changes during the aging process, e.g., deep tendon reflexes decrease, as proprioception does. In contrast, polyneuropathies have to be distinguished from age-associated changes as independent diseases with etiologies similar to those in younger ages. Incidence of polyneuropathies is reported about $118 / 100,000$, the overall prevalence in the general population is estimated to be about $1 \%$ and rises to up to $7 \%$ in the elderly. Etiology includes metabolic disorders, primary inflammatory polyneuropathies, systemic disorders and vasculitic neuropathies. Due to the age-specific increase of the prevalence of certain etiologies, neuropathies associated with diabetes, malignancy, and monoclonal gammopathies are even more common in older patients. However, the proportion of cryptogenic neuropathies, e.g. neuropathies without obvious cause, increases also with age. In older age, polyneuropathies additionally impair mobility and increase the risk of falling, thus the assessment of functional abilities is mandatory. It is essential to try to identify the underlying cause by a systematic approach including history, clinical investigation, neurophysiological and lab exams. Treatment of polyneuropathies is based on therapy of underlying conditions and requires management of neuropathic pain in the majority of cases. Physiotherapy and rehabilitation target pain relief and sustaining activities of daily living.

\section{Keywords}

Aging · Peripheral nervous system · Polyneuropathy, axonal, idiopathic, chronic · Diabetic neuropathies - Monoclonal gammopathy of undetermined significance

\section{- einer chronischen inflammatorischen demyelinisierenden Polyradikulo- neuropathie (CIDP) und \\ - einer längenabhängigen symmetri- schen axonalen Neuropathie (LDN).}

Die DADS-Neuropathie ist eine distale sensibel betonte Neuropathie mit deutlicher Ataxie und Tremor. Obwohl sie nur langsam progredient ist, kann es nach jahrelanger Erkrankung zu deutli- chen Behinderungen kommen [30]. Der typische elektrophysiologische Befund ist der einer distalen Demyelinisierung in der motorischen Neurographie, ein Befund der sich mit dem TerminaleLatenz-Index quantifizieren lässt [6].

Bei der DADS-Neuropathie findet sich in der Regel eine IgM-MGUS und in etwa $60 \%$ der Fälle ein Antikörper gegen myelinassoziiertes Glykoprotein (MAG). Eine CIDP, eine motorische und 
sensible, distale und proximale, symmetrische progrediente Neuropathie, können mit einer IgM-, ImmunglobulinA(IgA)- und Immunglobulin-G(IgG)MGUS vergesellschaftet sein. Bei der normalerweise anfangs milden und nur langsam progredienten LDN bei IgGund IgA-MGUS ist die Kausalität zwischen MGUS und Neuropathie um einiges unklarer als bei den anderen Formen [30-32]. Selten führt jedoch eine IgA- oder IgG-MGUS zu einer Leichtketten(AL)-Amyloidose. Daran sollte bei rasch progredienten, meist axonalen Neuropathien, Gewichtsverlust, Organomegalie, Kardiomyopathie und Makroglossie gedacht werden. Eine Biopsie, idealerweise von einem betroffenen Organ, hilft die Diagnose zu sichern [31, 32]. Eine Rarität ist die CANOMAD - eine chronische ataktische Neuropathie mit Ophthalmoplegie, M-Gradienten, Kälteagglutininen und Antikörpern gegen Disialosylganglioside $[25,46]$.

\section{》) Trotz langsamer Progredienz kann die DADS-Neuropathie nach Jahren zu deutlichen Behinderungen führen}

Die Therapie der CIDP mit IgA- oder IgG-MGUS entspricht der der idiopathischen CIDP [20]. In Fällen einer Neuropathie bei IgM-MGUS besteht keine Evidenz, dass eine immunsuppressive Therapie wirksam ist [20]. In progredienten Fällen ist der Versuch einer immunmodulierenden oder-supprimierenden Therapie, beispielsweise mit Steroiden, intravenösen Immunglobulinen oder Plasmaseparation, gerechtfertigt. Obwohl eine Studie zur Behandlung der MAG-Neuropathie mit Rituximab negativ war [19, 23], wird es im Alltag doch immer wieder auch erfolgreich eingesetzt [38].

\section{Polyneuropathien durch Vitaminmangel}

Eine Mangelernährung als Ursache von Hypovitaminosen findet sich im Alter bei gestörter enteraler Resorption, meist besteht ein Mangel an B-Vitaminen. Ein
Mangel an Vitamin $\mathrm{B}_{1}$ (Thiamin), $\mathrm{B}_{6}$ (Pyridoxin) oder auch $\mathrm{B}_{12}$ (Cobalamin) kann zu Neuropathien führen [36]. Am häufigsten ist der Vitamin- $B_{12}$-Mangel, der sich bei bis zu $6 \%$ der $<60$-Jährigen und bei bis zu $20 \%$ der $>60$-Jährigen findet [18]. Typisches klinisches Bild ist die Kombination von Rückenmarkssymptomen und Symptomen einer PNP [36]. Ein klinischer Hinweis auf einen Vitamin- $\mathrm{B}_{12}$-Mangel ist das gleichzeitige Auftreten von sensiblen Symptomen an Händen und Füßen. Die isolierte Bestimmung von Vitamin $B_{12}$ reicht nicht aus, da ein funktioneller Vitamin- $B_{12}$-Mangel auch bei niedrig-normalen Vitamin- $B_{12}-$ Werten beobachtet wurde. Die Bestimmung von Homocystein und vor allem Methylmalonat erhöht die diagnostische Sensitivität beträchtlich $[18,36]$. Bei einem Mangel sollte eine sofortige parenterale Substitution erfolgen, da eine Besserung in der Regel nur bei sehr frühem Therapiebeginn zu erwarten ist [36].

Neuere Beobachtungen legen eine Assoziation von L-Dihydroxyphenylalanin (L-DOPA) mit Morbus Parkinson und PNP nahe. Es hat sich gezeigt, dass Höhe und Dauer der L-DOPA-Dosierung mit Hinweisen auf einen Vitamin- $B_{12}$-Mangel und auch mit der Wahrscheinlichkeit einer Neuropathie korrelieren [7]. Daher sollte vor allem bei Patienten mit Morbus Parkinson, Langzeit-L-DOPA-Therapie und PNP an einen Vitamin- $\mathrm{B}_{12-}$ Mangel gedacht werden [33].

\section{Paraneoplastische Neuropathien}

Zwei Drittel aller malignen Erkrankungen treten jenseits des 65. Lebensjahrs auf. Das PNS kann dabei in unterschiedlicher Form involviert sein. Eine lokale Tumorinfiltration kann Hirnnerven, einzelne oder mehrere Nervenwurzeln im Plexus brachialis oder lumbosacralis sowie periphere Nerven treffen. Antineoplastische Therapeutika können Nebenwirkungen entfalten, die periphere Nerven schädigen [36]. In seltenen Fällen geht eine Neuropathie als paraneoplastisches Syndrom der Entdeckung einer Tumorerkrankung voraus [3]. Häufigste Ursache ist das kleinzellige Bronchialkarzinom, gefolgt von Mamma- und
Ovarialkarzinomen sowie Lymphomen. Die typische Präsentation ist die einer subakuten sensiblen Neuronopathie mit multifokalem und asymmetrischem Beginn. Früh sind die Arme betroffen, oft auch Gesicht und Rumpf. Häufig treten neuropathische Schmerzen auf, ebenso eine ausgeprägte sensible Ataxie der oberen und unteren Extremitäten. Bei mehr als $90 \%$ der Patienten können im Serum antineuronale Antikörper nachgewiesen werden, am häufigsten Anti-Hu oder Anti-CV2/CRMP5. Immunmodulation und symptomatische Therapieformen ergänzen das Spektrum [3].

\section{Hereditäre Transthyretin- amyloidose}

Trotz der Seltenheit muss die hereditäre Transthyretinamyloidose (hATTRAmyloidose) erwähnt werden, da kausale Therapiemöglichkeiten zur Verfügung stehen. Die hATTR-Amyloidose ist eine sehr seltene Multisystemerkrankung, die autosomal-dominant, aber mit variabler Penetranz vererbt wird [13]. Neben einer früh beginnenden gibt es auch eine spät, zwischen dem 50. und 70. Lebensjahr, einsetzende Form.

Neben einer kardialen Form mit Kardiomyopathie präsentiert sich die hATTR-Amyloidose häufig in Form einer Neuropathie, der familiären Amyloidneuropathie (TTR-FAP; [2]). Typisch ist eine ungewöhnlich rasch progrediente, meist axonale Neuropathie. „Red flags" für die Diagnose sind deutliche Gangunsicherheit, Gewichtsverlust, autonome Beteiligung, Nierenbeteiligung und bei Älteren eine beidseitige Operation wegen eines Karpaltunnelsyndroms in der Anamnese [9]. Die TTR-FAP wird in 3 Stadien eingeteilt [1]:

- Stadium 1: Gehen ohne fremde Hilfe - Stadium 2: Gehen mit fremder Hilfe

- Stadium 3: Rollstuhlpflicht oder

Bettlägerigkeit

Die Diagnose kann durch Biopsie des betroffenen Gewebes bestätigt werden, allerdings bestehen etliche Unsicherheiten hinsichtlich der Gewebeauswahl sowie histologischen und immunhistologischen Aufarbeitung [1]. Aus diesem 
Grund rückt die genetische Diagnostik zunehmend in den Vordergrund.

Da die Erkrankung rasch progredient verläuft, sind eine frühe Diagnose und Therapie entscheidend.ZurTherapie sind derzeit drei Substanzen zugelassen: Tafamidis, ein TTR-Stabilisator; Patisiran, eine in Lipidnanopartikel verpackte „small interfering RNA“, und Inotersen, ein Antisense-Oligonukleotid [1]. Tafamidis ist nur für die Behandlung im Stadium 1 zugelassen, Patisiran und Inotersen im Stadium 1 und 2.

\section{Therapie}

Die allgemeinen Prinzipien der Therapie von Neuropathien werden in einem anderen Beitrag dieses Schwerpunkts von Sachau et al. beschrieben. Neben der Behandlung der Grunderkrankung erweist sich im Alter die Behandlung des neuropathischen Schmerzes oft als komplex und herausfordernd, Studiendaten für alte Patienten stehen kaum zur Verfügung. Dabei sind alte Menschen deutlich vulnerabler als junge, da sie häufig von Multimorbidität, Mangelernährung, Sarkopenie und Gebrechlichkeit („,frailty") betroffen sind. Organinsuffizienzen und Multimedikation tragen zu einem höheren Risiko für unerwünschte Arzneimittelwirkungen bei [10]. Bis zum 80. Lebensjahr reduzieren sich beim Organgesunden die Funktionen von Niere und Leber um bis zu $40 \%$ mit entsprechenden Folgen für Metabolisierung und Elimination vieler Arzneimittel. Zudem werden Kompensationsmechanismen zunehmend eingeschränkt, sodass $\mathrm{Ne}$ benwirkungen auf das Herz-KreislaufSystem und das zentrale Nervensystem, beispielsweise in Form von Schwindel und Mattigkeit, erhöhtem Sturzrisiko oder Benommenheit, häufiger werden.

Eine eingeschränkte Nierenfunktion spielt eine besondere Rolle in der Therapie mit Gabapentin und Pregabalin, wo eine entsprechende Erniedrigung der maximalen Tagesdosis zu beachten ist. Die Anwendung von Carbamazepin und Oxcarbazepin ist durch das Risiko von Hyponatriämie und Arzneimittelinteraktionen limitiert [29].

Trizyklische Antidepressiva sind im Alter aufgrund ihres anticholinergen
Nebenwirkungsprofils nur mit äußerster Zurückhaltung und in niedrigen Dosierungen einzusetzen. Anticholinerge Nebeneffekte können negative Auswirkungen auf die kognitiven Fähigkeiten haben, Delirien auslösen, aber auch durch Mundtrockenheit zu einer verminderten Nahrungs- und Flüssigkeitsaufnahme beitragen.

\section{》) Trizyklische Antidepressiva werden im Alter nur mit äußerster Zurückhaltung eingesetzt}

Bei der Therapie mit Serotonin-Noradrenalin-Wiederaufnahmehemmern (Duloxetin, Venlafaxin) und selektiven Serotoninwiederaufnahmehemmern (Citalopram, Paroxetin) ist das besonders bei Frauen erhöhte Risiko einer Hyponatriämie zu erwähnen, bei Multimedikation ist auch die QT-Zeit zu kontrollieren. Überdies ist die Gabe von Antidepressiva im Alter mit einem erhöhten Sturzrisiko verbunden [35].

Bei Opiattherapie ist ein erhöhtes $\mathrm{Ne}$ benwirkungsrisiko seitens des zentralen Nervensystems gegeben, Verschlechterungen der kognitiven Funktionen bis hin zum Delir sind möglich. Daneben ist die Prophylaxe einer Obstipation zu gewährleisten. Die topische Anwendung von Capsaicin ist häufig durch die Fragilität der Haut limitiert.

Die Anwendung physikalischer Methoden ergänzt das therapeutische Spektrum. Besondere Bedeutung kommt dem Training von Gleichgewicht und Kraft im Sinne einer Sturzprophylaxe zu.

Generell ist festzuhalten, dass es zur Therapie der PNP im höheren Lebensalter wenig Literatur gibt, es ist aber davon auszugehen, dass die aktuellen Leitlinienempfehlungen mit den erwähnten Einschränkungen auch für die Therapie alter Menschen herangezogen werden können.

\section{Fazit für die Praxis}
- Die Polyneuropathie (PNP) ist vor- wiegend eine Erkrankung des alten Menschen.
- In der Diagnostik müssen die al- tersspezifischen Veränderungen des

peripheren Nervensystems, die den klinischen Befunden bei Neuropathien ähneln können, berücksichtigt werden.

- Die Abklärung der möglichen Ursachen sollte sich am Phänotyp der Neuropathie orientieren, wobei die Kenntnis der seltenen, aber behandelbaren Phänotypen eine entscheidende Rolle spielt. Am häufigsten ist allerdings die mild verlaufende chronische idiopathische axonale PNP ohne fassbare Ätiologie.

\section{Korrespondenzadresse}

\section{W. N. Löscher}

Universitätsklinik für Neurologie, Medizinische Universität Innsbruck

Anichstr. 35, 6020 Innsbruck, Österreich wolfgang.loescher@i-med.ac.at

Funding. Open access funding provided by University of Innsbruck and Medical University of Innsbruck.

\section{Einhaltung ethischer Richtlinien}

Interessenkonflikt. W.N. Löscher und B. Iglseder geben an, dass kein Interessenkonflikt besteht.

Für diesen Beitrag wurden von den Autoren keine Studien an Menschen oder Tieren durchgeführt. Für die aufgeführten Studien gelten die jeweils dort angegebenen ethischen Richtlinien.

Open Access. Dieser Artikel wird unter der Creative Commons Namensnennung 4.0 International Lizenz veröffentlicht, welche die Nutzung, Vervielfältigung, Bearbeitung, Verbreitung und Wiedergabe in jeglichem Medium und Format erlaubt, sofern Sie den/die ursprünglichen Autor(en) und die Quelle ordnungsgemäß nennen, einen Link zur Creative Commons Lizenz beifügen und angeben, ob Änderungen vorgenommen wurden.

Die in diesem Artikel enthaltenen Bilder und sonstiges Drittmaterial unterliegen ebenfalls der genannten Creative Commons Lizenz, sofern sich aus der Abbildungslegende nichts anderes ergibt. Sofern das betreffende Material nicht unter der genannten Creative Commons Lizenz steht und die betreffende Handlung nicht nach gesetzlichen Vorschriften erlaubt ist, ist für die oben aufgeführten Weiterverwendungen des Materials die Einwilligung des jeweiligen Rechteinhabers einzuholen.

Weitere Details zur Lizenz entnehmen Sie bitte der Lizenzinformation auf http://creativecommons.org/ licenses/by/4.0/deed.de. 


\section{Literatur}

1. Adams D, Koike H, Slama M, Coelho T (2019) Hereditary transthyretin amyloidosis: a model of medical progress for a fatal disease. Nat Rev Neurol 15:387-404. https://doi.org/10.1038/ s41582-019-0210-4

2. Ando Y, Coelho T, Berk JL et al (2013) Guideline of transthyretin-related hereditary amyloidosis for clinicians. Orphanet J Rare Dis 8:31. https://doi. org/10.1186/1750-1172-8-31

3. Antoine J-C, Camdessanché J-P (2017) Paraneoplastic neuropathies. Curr Opin Neurol 30:513-520. https://doi.org/10.1097/WCO. 0000000000000475

4. Arnold N, Harriman DG (1970) The incidence of abnormality in control human peripheral nerves studied by single axon dissection. J Neurol Neurosurg Psychiatr 33:55-61. https://doi.org/10. 1136/jnnp.33.1.55

5. Callaghan B, Kerber K, Langa KM et al (2015) Longitudinal patient-oriented outcomes in neuropathy: importance of early detection and falls. Neurology 85:71-79. https://doi.org/10.1212/ WNL.0000000000001714

6. Capasso M, Torrieri F, Di Muzio A et al (2002) Can electrophysiology differentiate polyneuropathy with anti-MAG/SGPG antibodies from chronic inflammatory demyelinating polyneuropathy? Clin Neurophysiol 113:346-353

7. Ceravolo R, Cossu G, Bandettini Di Poggio M et al (2013) Neuropathy and levodopa in Parkinson's disease: evidence from a multicenter study. Mov Disord 28:1391-1397. https://doi.org/10.1002/ mds. 25585

8. Clark BC, Manini TM (2012) What is dynapenia? Nutrition 28:495-503. https://doi.org/10.1016/j. nut.2011.12.002

9. Conceição I, González-Duarte A, Obici L et al (2016) "Red-flag" symptom clusters in transthyretin familial amyloid polyneuropathy. J Peripher Nerv Syst 21:5-9. https://doi.org/10.1111/jns.12153

10. Davies EA, O'Mahony MS (2015) Adverse drug reactions in special populations - the elderly. Brit J Clin Pharmacol 80(4):796-807. https://doi.org/10. 1111/bcp.12596

11. Dyck PJB, Thaisetthawatkul P (2014) Lumbosacral plexopathy. Continuum 20:1343-1358. https:// doi.org/10.1212/01.CON.0000455877.60932.d3

12. Dyck PJB, Windebank AJ (2002) Diabetic and nondiabetic lumbosacral radiculoplexus neuropathies: new insights into pathophysiology and treatment. Muscle Nerve 25:477-491

13. Finsterer J, Iglseder S, Wanschitz J et al (2018) Hereditary transthyretin-related amyloidosis. Acta Neurol Scand 86:1036. https://doi.org/10.1111/ ane. 13035

14. Goble DJ, Coxon JP, Wenderoth N et al (2009) Proprioceptive sensibility in the elderly: degeneration, functional consequences and plastic-adaptive processes. Neurosci Biobehav Rev 33:271-278. https://doi.org/10.1016/j.neubiorev.2008.08.012

15. Hanewinckel $R$, Drenthen J, van Oijen $M$ et al (2016) Prevalence of polyneuropathy in the general middle-aged and elderly population. Neurology 87:1892-1898. https://doi.org/10. 1212/WNL.0000000000003293

16. Hanewinckel R, Drenthen J, Verlinden VJA et al (2017) Polyneuropathy relates to impairment in daily activities, worse gait, and fall-related injuries. Neurology 89:76-83. https://doi.org/10.1212/ WNL.0000000000004067

17. Hanewinckel R, van Oijen M, Ikram MA, Van Doorn PA (2016) The epidemiology and risk factors of chronic polyneuropathy. Eur J Epidemiol 31:5-20. https://doi.org/10.1007/s10654-0150094-6

18. Hunt A, Harrington D, Robinson S (2014) Vitamin B12 deficiency. BMJ 349:g5226-g5226. https:// doi.org/10.1136/bmj.g5226

19. lancu Ferfoglia R, Guimarães-Costa R, Viala $K$ et al (2016) Long-term efficacy of rituximab in lgM anti-myelin-associated glycoprotein neuropathy: RIMAG follow-up study. J Peripher Nerv Syst 21:10-14. https://doi.org/10.1111/jns.12156

20. Joint Task Force of the EFNS and the PNS (2010) European Federation of Neurological Societies/Peripheral Nerve Society Guideline on management of paraproteinemic demyelinating neuropathies. Report of a Joint Task Force of the European Federation of Neurological Societies and the Peripheral Nerve Society-first revision. J Peripher Nerv Syst 15:185-195. https://doi.org/ 10.1111/j.1529-8027.2010.00278.x

21. Katzberg HD, Kassardjian CD (2016) Toxic and endocrine myopathies. Continuum 22:1815-1828. https://doi.org/10.1212/CON.0000000000000407

22. Kyle RA, Larson DR, Therneau TM et al (2018) Long-term follow-up of monoclonal gammopathy of undetermined significance. $\mathrm{N}$ Engl J Med 378:241-249. https://doi.org/10.1056/ NEJMoa1709974

23. Léger J-M, Viala K, Nicolas G et al (2013) Placebocontrolled trial of rituximab in IgM anti-myelinassociated glycoprotein neuropathy. Neurology 80:2217-2225. https://doi.org/10.1212/WNL. Ob013e318296e92b

24. Low PA, Dyck PJ (1978) Splanchnic preganglionic neurons in man. III. Morphometry of myelinated fibers of rami communicantes. J Neuropathol Exp Neurol 37:734-740. https://doi.org/10.1097/ 00005072-197811000-00002

25. Löscher W, Woertz A, Wallnöfer M et al (2013) Successful treatment of CANOMAD with IVIg and rituximab. J Neurol 260:1168-1170. https://doi. org/10.1007/s00415-013-6867-5

26. Martina IS, Van Koningsveld R, Schmitz PI et al (1998) Measuring vibration threshold with a graduated tuning fork in normal aging and in patients with polyneuropathy. European Inflammatory Neuropathy Cause and Treatment (INCAT) group. J Neurol Neurosurg Psychiatry 65:743-747

27. Mathis S, Vallat JM, Ingrand P et al (2015) Causes of neuropathy in the elderly: a retrospective study with 785 patients. Eur Geriatr Med 6:114-118. https://doi.org/10.1016/j.eurger.2015.02.006

28. Oppenheim (1893) Über die senile Form der Polyneuritis. Berl Klin Wochenschr 25

29. Perucca E, Berlowitz D, Birnbaum A et al (2006) Pharmacological and clinical aspects of antiepileptic drug use in the elderly. Epilepsy Res 68(Suppl 1):S49-S63. https://doi.org/10.1016/j.eplepsyres. 2005.07.017

30. Raheja D, Specht C, Simmons Z (2015) Paraproteinemic neuropathies. Muscle Nerve 51:1-13. https://doi.org/10.1002/mus.24471

31. Rajabally YA (2011) Neuropathy and paraproteins: review of a complex association. Eur J Neurol 18:1291-1298. https://doi.org/10.1111/j.14681331.2011.03380.x

32. Rison RA, Beydoun SR (2016) Paraproteinemic neuropathy: a practical review. BMC Neurol 16:13-14. https://doi.org/10.1186/s12883-0160532-4

33. Romagnolo A, Merola A, Artusi CA et al (2019) Levodopa-induced neuropathy: a systematic review. Mov Disord Clin Pract 6:96-103. https:// doi.org/10.1002/mdc3.12688

34. Singer MA, Vernino SA, Wolfe GI (2012) Idiopathic neuropathy: new paradigms, new promise. JPeripher Nerv Syst 17(Suppl 2):43-49. https://doi. org/10.1111/j.1529-8027.2012.00395.x

35. Sobieraj DM, Martinez BK, Hernandez AV et al (2019) Adverse effects of pharmacologic treatments of major depression in older adults. J Am Geriatr Soc 67:1571-1581. https://doi.org/10. $1111 /$ jgs. 15966

36. Staff NP, Windebank AJ (2014) Peripheral neuropathy due to vitamin deficiency, toxins, and medications. Continuum 20:1293-1306. https:// doi.org/10.1212/01.CON.0000455880.06675.5a

37. Suetterlin KJ, Sayer AA (2014) Proprioception: where are we now? A commentary on clinical assessment, changes across the life course, functional implications and future interventions. Age Ageing 43:313-318. https://doi.org/10.1093/ ageing/aft174

38. Svahn J, Petiot P, Antoine J-C et al (2018) Anti-MAG antibodies in 202 patients: clinicopathological and therapeutic features. J Neurol Neurosurg Psychiatry 89:499-505. https://doi.org/10.1136/ jnnp-2017-316715

39. Taylor PK (1984) Non-linear effects of age on nerve conduction in adults. J Neurol Sci 66:223-234. https://doi.org/10.1016/0022-510x(84)90011-x

40. van Koningsveld R, Steyerberg EW, Hughes RAC et al (2007) A clinical prognostic scoring system for Guillain-Barré syndrome. Lancet Neurol 6:589-594. https://doi.org/10.1016/S14744422(07)70130-8

41. Visser NA, Notermans NC, Linssen RSN et al (2015) Incidence of polyneuropathy in Utrecht, the Netherlands. Neurology 84:259-264. https://doi. org/10.1212/WNL.0000000000001160

42. Visser NA, Vrancken AFJE, van der Schouw YT et al (2013) Chronic idiopathic axonal polyneuropathy is associated with the metabolic syndrome. Diabetes Care 36:817-822. https://doi.org/10. 2337/dc12-0469

43. Vrancken AFJE, Franssen $\mathrm{H}$, Wokke JHJ et al (2002) Chronic idiopathic axonal polyneuropathy and successful aging of the peripheral nervous system in elderly people. Arch Neurol 59:533-540

44. Vrancken AFJE, Kalmijn S, Brugman F et al (2006) The meaning of distal sensory loss and absent ankle reflexes in relation to age: a meta-analysis. J Neurol 253:578-589. https://doi.org/10.1007/ s00415-005-0064-0

45. Vrancken AFJE, Notermans NC, Wokke JHJ, Franssen H (2008) The realistic yield of lower leg SNAP amplitudes and SRAR in the routine evaluation of chronic axonal polyneuropathies. J Neurol 255:1127-1135. https://doi.org/10.1007/ s00415-008-0817-7

46. Willison HJ, O'Leary CP, Veitch J et al (2001) The clinical and laboratory features of chronic sensory ataxic neuropathy with anti-disialosyl lgM antibodies. Brain 124:1968-1977

47. Zis P, Sarrigiannis PG, Rao DG et al (2016) Chronic idiopathic axonal polyneuropathy: a systematic review. J Neurol 263:1903-1910. https://doi.org/ 10.1007/s00415-016-8082-7 